\title{
Chemische Untersuchung der Mineralwasser von Cheltenham;
}

\author{
von F. A. Abel und T. H. Rowney,
}

Assistenten in Royal College of Chemistry London.

(Gelesen vor der "Chemical Socicly of London" im Mai 1848.)

In den folgenden Analysen liefern wir einen Beitrag zu einer umfassenden Unlersuchung sämmtlicher Mineralwasser in Grolsbriltanien, welche auf Veranlassung des Herrn Dr. Hof$m a n n$ in dem hiesigen Laboratorium begonnen worden ist.

Die vorliegenden Analysen schliefsen sich, was die Methode anlangl, genau an die bereits früher von den Herren Merck und G all ow a y veröffentlichte Untersuchung des Mineralwassers von Balh.

Die Mineralquellen von Cheltenham, deren gegenwärlig vierzehn im Gebrauch, sind über die ganze Stadt und deren Ungebung verbreilet. Der Bequemlichkeit halber werden dic Wasser aber von den Eigenthümern miltelst eiserner Röhren nach verschiedenen, in der Nähe der Hauptquellen errichteten Kursälen hingefülrt. Da sich bei Untersuchung an Ort und Stelle ergab, dafs viele dieser Wasser in ihrer Zusammenselzung nur wenig von einander abwichen, so würde es Zeitverschwendung gewesen seyn, sie alle der Analyse zu unterwerfen; so beschlossen wir, nur die für die Unlersuchung zu wählen, welche als Repräsentanten der übrigen angesehen werden könnell. Unter diesen ist das Wasser von Montpelier Spa erst vor Kurzem von Herrn S. 'T. Co op er analysirt worden; unter den übrigen mufste sich unscre Wahl vorzugsweise denjenigen Quellen zulenken, welche entweder noch gar nicht, oder nur vor sehr langer Zeit untersucht worden sind. 
Die folgende Analyse umfarst die eisenhaltige Quelle von Cambray, die zwei Hauptwasser der Royal Old Wells und die sogenannte starke Salzquelle von Pitville. Eine Analyse der letzleren schien uns besonders wïnschenswerth, indem die von Daniel, Daubeny und Buckmann zu verschiedenen Zeiten veröffentlichten Analysen derselben so wenig Uebereinstimmung zeigen. Wir schöpflen das Wasser an Ort und Stelle auf die gewöhnliche Art; die einzige Schwierigkeit bestand in dem Auffangen des zur Bestimmung der Kohlensüure nöthigen Wassers. Die grolse Tiefe und der geringe Durchmesser der Brunnen machten es unmöglich, hinabzusteigen, um einen Stechheber in dic Quelle einzusenken, auch verhinderten die alle Brunnen durchkreuzenden dicken Querbalken das Hinablassen eines grofsen Schöpfgefäfses; wir muIsten uns daher darnil begnügen, den calibrirten Heber an der Mündung der Pumpen zu füllen. Da diese nur langsam in Bewegung gesetzt wurden und das Wasser aus der Tiefe der Brunnen schöpften, glaublen wir nicht, einen grofsen Verlust an Kohlensäure befürchten zu müssen. Directe Versuche, welche wir früher bei einer Analyse des artesischen Brunnens in 'Trafalgar Square *) anstellten, haben gezeigt, dafs die durch Pumpen verlorene Kohlensäurc aufserordentlich gering ist.

\section{Eisenhallige Quelle von Cambray.}

Diese Quelle bei Cambray, auf der Nordseite des Flusses Chelt gelegen, ist bereits seit 1807 im Gebrauch. Sie befindet sich unter dem Fufsboden der Küche in dem Wohnhause des Eigenthümers, von wo das Wasser miltelst eiserner Rölren über den kleinen Flufs Chelt nach dem ungefähr 100 Schritte von der Quelle liegenden Kursaale geleitet wird. Die 'Tiefe des

*) Quarterly Journal of the Chemical Society of London. 
Brunnens ist etwa 7 Fufs. Als wir das Wasser schöpften, war die Temperalur desselben $18^{\circ} \mathrm{C}$, die der Almosphäre $25^{\circ} \mathrm{C}$. Das Wasser reagirt alkalisch, riecht schwach nach Schwefelwasserstoffsäure und besilzt aufserdem einen eigenlhiumlichen Geruch nach vermodernden Pflanzenstoffen. Das specifische Gewicht ist bei $16,5^{\circ}$ C. 1,001. Beim Ausflusse aus der Pumpe ist das Wasser ganz klar, wird aber fast augenblicklich trübe, selbst in verschlossenen Gefälsen und setzt eine Quantilät Eisenoxyd $a b$, womit auch alle zum Trinken benutzte Gläser bedeckt sind. Der qualitativen Analyse unterworfen, wurden Schwefelsäure, Chlor, Phosphorsäure, Kieselsäure und Kohlensäure; Eisenoxyd, Kalk, Magnesia, Kali, Natron und organische Materie vorgefunden. Brom und Jod waren nicht zu entdecken. Ein Theil des Natrons existirt als kohlensaures Salz.

\section{Salzquelle von Pitville.}

Diese Quelle wird aus fünf Brunnen gespeist, die sich unter dem Kursaale und in dessen Nälie befinden und aus welchen sich das Wasser in dem 90 Fufs tiefen Hauptbrunnen sammelt. Die Temperatur des Wassers ist $19,5^{\circ} \mathrm{C}$. bei $14^{\circ} \mathrm{C}$. Lufttemperatur. Es besilzt eine starke alkalische Reaclion, einen sehr schwachen Geruch nach Schwefelwasserstoffsäure; einen starken, aber angenehmen salinischen Geschmack und ein specifisches Gewicht von 1,00763 bei $15,5^{\circ} \mathrm{C}$. Die bei qualitativer Analyse gefundenen Bestandtheile stimmten mit denen des vorigen $W$ assers überein, ausgenommen, dafs keine Spur Eisen, dagegen aber eine bestimmbare Quantităt Brom und deulliche Spuren von Jod vorhanden sind.

\section{IIl. Schoefelhaltiges Salzwasser (No. I.) (Royal Old Wells).}

An dieser Quelle sollen die medicinischen Eigenschafien der Cheltenhamer Wasser gegen 1706 zuerst beobachtet worden 
seyn. Als Veranlassung werden Taubenflüge angegeben, wclche sich gelegentlich an der Quelle eines auf der Südseite der Stadt gclegenen Baches niederlielsen, um die Salzkörner aufzupicken, welche das in der Sonnenhitze verdampfende Wasser zurückliefs. Die ursprüngliche Quelle war etwa 6 Fufs unter der Erdoberfäche. Im Jahre 1808 wurde ein Schacht von 12 Fufs Tiefe und 6 Fuls Durchmesser gegraben, der 1819 bis auf 72 Fufs verlieft wurde, welches auch die gegenwärlige Ticfe des Brunnens ist. Die Temperatur des Wassers war $14^{\circ} \mathrm{C}$, bei einer Luftemperatur von $22^{\circ} \mathrm{C}$. Es reagirt scliwach alkalisch und besilzt einen reinsalzigen Gesclimack. Obgleich der Geruch nach Schwefelwasserstoffsäure in diesem Wasser weit stärker war, wie in irgend einem der andern von uns analysirten, so liefs sich dic Quantilät dieses Gases dennoch nicht bestimmen. Das specilische Gewicht des Wassers ist bei $15,5^{\circ}$ C. 1,0064 . Aufser den in dem Pitviller Wasser vorgefundenen Bestandtheilen wurde noch Eisen als phosphorsaures und kohlensaures Salz nachgewiesen. Es waren nur Spuren von Kali und Jod vorhanden; ein Theil des Kalks und der Bittererde befindel sich in dem Wasser in der Form löslicher Salze, die nicht durch Kochen gefällt werden.

\section{Stavke Salsquelle (No. 1.) \\ (Royal Old Wells).}

Diese Quelle ist nicht weit von der vorigen enlfernt. Die Tiefe dieses Brunnens ist 71 Fufs; die Höhe des Wasserstandes war bei unserer Anwesenheit in Cheltenham 12 Fufs. Das Wasser, dessen Zuflufs nicht so regelmälsig ist, wie bei den anderen, wird mittelst eiserner Röhren nach dem Hauplsaale geleitet. Es kann aber auch von Patienten in einem ganz in der Nähe des Brunnens gelegenen Zimmer getrunken werden. Das zur Analyse verwendete Wasser wurde von diesem Locale genommen. Die 'Temperalur desselben war $14^{\circ} \mathrm{C}$, , bei einer 
Luftemperalur von $22^{\circ} \mathrm{C}$; es hat eine schwach alkalisclie Reaction, einen salinischen Geschmack und einen schwachen Geruch nach Schwefelwasserstoftsäure und enthält die nämlichen Bestandtheile, wie das vorhergehende Wasser. Das Jod in diesem Wasser ist bestinmbar, von Eisen und Phosphorsäure aber sind nur Spuren vorhanden.

Die Details der quantilativen Analyse dieser Quellen haben wir in dem Journal of the Chemical Society of London (October 1848) mitgetheilt. Aus diesen Analysen ergiebt sich folgende Zusammenselzung : 


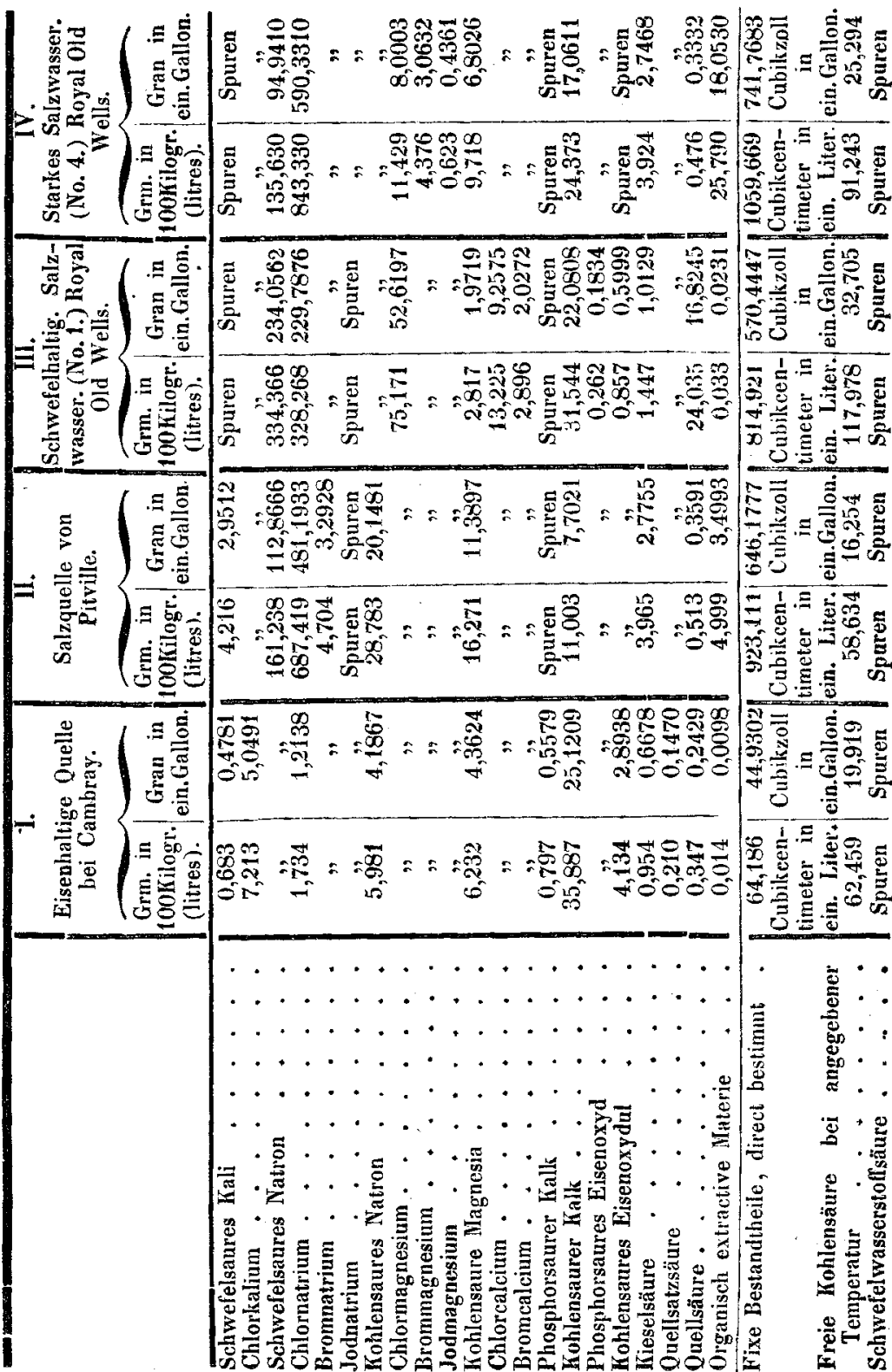


Bei der Berechnung der obigen Resultate ergiebt sich, dafs der Kalk und die Biltererde, welche durch Sieden nicht gefällt wurden, nichisdesloweniger als kohlensaure Salze vörhanden seyn mufsten, welche von den alkalischen Salzen in Lösung gehalten wurden. Es ist bekannt, dafs kohlensaurer Kalk nicht vollkommen unlöslich in Wasser ist, 1 Theil kohlensaurer Kalk bedarf ungefähr 10000 Theile Wasser zur Lösung; ferner, dafs die Löslichkeit durch Gegenwart von Ammoniak oder kohlensauren Ammoniak vermehrt wird (1 Theil bedarf nur ungefähr 6800 Theile ammoniakalischen Wassers). Wir haben uns durch Versuche überzeugt, dafs die Löslichkeit des kohlensauren Kalks in Wasser durch Gegenwart von Chlornatrium und schwefelsaures Natron ebenfalls vermehrt wird; in einer solchen Lösung entsteht nach Ausfällung des Kalkes mittelst kohlensauren Ammoniaks durch oxalsaures Ammoniak noch ein bemerkbarer Niederschlag, während in reinem oder ammoniakalischem Wasser nur eine kaum bemerkbare Trübung erscheint. Diese geringe Löslichkeit des kohlensauren Kalkes in alkalischen Salzen machte es für die genaue Bestimmung der Alkalien erforderlich, nach dem Fällen mit kohlensaurem Ammoniak ein Paar Tropfen oxalsaures Ammoniak hinzuzufügen, zur Ausfällung der letzten Spuren von Kalk oder von Baryt, welches zur Scheidung der Bittererde gedient hat.

Bei einer Betrachtung der in der Tabelle angefuhrten Resullate erscheint vor Allem die kleine Quantitat der in dem Wasser von Cambray enthaltenen festen Bestandtheile auffallend gering, im Vergleich mit dem Gehalt der anderen; während die drei leîzteren 800 bis $1050 \mathrm{Grm}$. in 100 Kilogrammen enthalten, finden wir in genanntem Wasser nur $45 \mathrm{Grm}$., welche kaum die in gewöhnlichem Brunnenwasser gefundene Quantilät übersteigt. Abgesehen von dem quantitativen Verhälinifs, ist auch die Natur der Bestandiheile sehr verschieden. Alle vier Wasser reagiren alkalisch, während aber diese Reaclion in dem 
Wasser von Cambray und Pilville, in Folge ansehnlicher Quanlitälen kohlensauren Natrons sehr deullich ist, wird sie bei den anderen kaum beinerkt und tritt erst bei Abdampfung bestimmler hervor, welches theilweise von der Zerselzung des in grolser Menge vorhandenen Chlormagnesiums herruihren mag. Die Quellen von Cambray und Pitville sind also alkalische, die anderen beiden salinische Säuerlinge.

Unsere Aualysen weichen bedeutend von allen anderen frïher veröffentlichten ab. In allen von uns untersuchten Wassern wurde Kali in gröfserer oder kleincrer Quantität entdeckt; in leiner anderen Analyse wird diefs erwähnt, aufser in einer von Scudamore, wo blofs angegeben wird, dafs Kali in kleiner Quantität in einem der Wasser der Royal Otd Wells vorhanden ist.

In einer Abhandlung von Daubeny *) über die Gegenwart von Jod und Brom in den Salzquellen Englands wird angegeben, dafs in keiner der Quellen von Cheltenham, welche Jod enthalten, Brom gefunden wurde, während in der Quelle von Pitville, der einzigen, welche kein Jod enthält, Brom vorhanden sey in dem Verhältnissc von 1 Gran zu 6 Gallonen Wasser. In drei der von uns analysirten Wasser wurde Brom in bestimmbarer Menge und stets von Jod begleitet, aufgefunden.

Auch die Gegenwart von Phosphorsäure ist bis jetat unbemerkt geblieben.

Was die gasförmigen Bestandtheile dieser Wasser anlangt, so ist die grofsc Quantität der vorhandenen Kohlensäure auffallend, besonders in der schwefelhaltigen Salzquelle; sie übersteigt bei wcitem den Kohlensäuregehalt der Thermalquelle von Bath.

Spuren von Schwelelwasserstoffsäure wurlen in allen Mineralwassern Cheltenhams vorgefunden, aber in keinem der von

*) Plilosophical Transactions. Mai 1830. 
uns analysirten Wasser war die Quantitit bestimmbar. In den vor einigen Jahren von Sc udamore *) veröffentlichten AnaIysen der Royal Old Wells wird die Quantität dieses Gases nicht nur als bestimmbar, sondern als sehr belrächllich angeführt. In seinem Werke über 'Thermalquellen **) sagt übrigens Dr. Gairdner, dals die Quantität der Schwefelwasserstoffsäure in diesem Wasser sehr unbeständig und bei nasser Jahreszeit slets grölser sey als sonst.

Die Bildung der Schwefelwasserstoftsäure rührt offenbar von der Zerselzung schwefelsaurer Salze durch organische Stoffe her. Mit Kork verstopfte Flaschen zeigten sich beim Oeffnen in London in hohem Grade schwefelwasserstoffhallig, während das Wasser in Flaschen mit Glasstöpseln nicht mehr von diesem Gase enthielt, als an der Quelle.

Die Mineralwasser von Cheltenham sind sehr veränderlich in Stärke und Constitution; es ist wohl bekannt, dafs manche Quellen, welche während einem gewissen Zeilraume in hohem Grade geschälzt gewvesen sind, plötzlich so viel ihrer Bestandtheile verlieren, dafs die Eigenthümer gezwungen sind, sie unbenutzt liegen zu lassen. Der Zuflufs des Wassers ist aus vielen Quellen schr beschränkt, wclches man schon daraus ersehen kann, dafs stels eine Anzahl von Brunnen gebohrt werden mufsten, um einen Hauplbrunnen ordentlich mit Wasser zu versehen. Der verstorbene Herr Thomson von Montpelier liefs eino grofse Anzahl Brunnen meilenweit in der Umgebung seines Kursaales bohren, um eine hinreichende Menge Wasser zu erhalten. Kostspielige Anstallen sind ferner von den verschiedenen Eigenlhümern getroflen worden, um dem Eindringen von Regenwasser in dic Quellen zu begegnen, eine

*) Chemisch-medicinischer Bericht über die Mineralwasser von Buxton, vou C. Scudamorc, M. D. 1820.

**) Thermal - und Mlineralquellen, von Dr. Gairdner. 1832. 
Thatsache, welche hinreichend ist, um den Argwohn zu entkrüftigen, dafs die Mineralwasser von Cheltenham von den Eigenthümern künsllich fabricirt werden; eine Meinung, welche vielleicht durch den Umstand vcranlafst worden ist, dafs sich dicht neben dem Monlpelier Kursaale ein Laboratorium befindet, wo das Wasser abgedampft wird, um die Salze zı crlalten, so wie dadurch, dafs sogenannles Chellenham-Salts künstlich in den Apolheken von Cheltenham und ganz England bereitet wird.

\section{Analyse eines Venensteins; \\ von J. Schlo/sberger.}

Durch Herrn Generalstabsarzt Dr. v. Klein erlielt ich vor einiger Zeit einen ächten Venenslein, von der Grölse einer kleinen Bolne, den derselbe bei der Section eines 22jährigen Mannes selbst aus der etwas erweiterten rechten Vena vesicalis, in welcher das Concrement frei gelegen, herausgenommen hatte. Da nur wenige chemische Untersuchungen solcher Concretionen vorhanden (von John, L. Gmelin, Lehmann, Nasse) und die vorhandenen auch zum Theil nur qualitaliver Art sind, so schien mir eine wiederholte genaue Analyse nicht olne einigen Werth.

Der Stein war länglich rund, von nicht selır deutlich geschichtetem Baue, in der Mitte eine klejne Höhlung zeigend. Die verschiedenen Schichten waren gelblich wcifs, brüchig; es konnte kein hautartiger Ueberzug als autserste Schichle nachgewiesen werden. Das Bindemittel schien eine eiweilsartige Substanz, wurde durch concentrirle Chlorwasserstoffsïure violetl, durch Essigsăure fast vollständig gelöst. 